\title{
A method for constructing the bilinear tension softening diagram of concrete corresponding to its true fracture energy
}

\author{
H. M. Abdalla* and B. L. Karihaloo* \\ Cardiff University
}

For the analysis of cracked concrete structures using the fictitious crack model two fracture properties of concrete are required, namely its true specific fracture energy $G_{\mathrm{F}}$ and the corresponding tension softening relation $\boldsymbol{\sigma}(w)$. In a recent paper, the authors proposed a simple method for the determination of the true specific fracture energy of a concrete mix. In this paper a method is proposed based on the concept of a non-linear hinge for constructing a bilinear approximation of the tension softening relation consistent with the true specific fracture energy $G_{\mathrm{F}}$. The parameters of this bilinear approximation are inferred in an inverse manner. It is shown that this inverse identification procedure predicts accurate bilinear softening relations of concrete mixes tested in three-point bend and wedge-splitting modes.

\section{Introduction}

In the analysis of cracked concrete structures the non-linear theory of fracture mechanics based on the fictitious crack model is often used. ${ }^{1}$ This model recognises the fact that an extensive fracture process zone (FPZ) exists ahead of a real traction-free crack in which concrete softens progressively due to microcracking. This tension softening FPZ is included in the model as a fictitious crack. The term 'fictitious' is used to underline the fact that this portion of the crack cannot be continuous with full separation of its faces, as in a real traction-free crack. The fictitious crack faces are able to transfer some stresses across them which are not constant over its length. In fact, they increase from nothing at the tip of a real traction-free crack to the full uniaxial tensile strength of concrete at the tip of the fictitious crack (Fig. 1). In the fictitious crack model (FCM) two material properties of concrete are needed in addition to its tensile strength $\boldsymbol{f}_{\mathrm{t}}$ and Young's modulus, E. These are the specific fracture energy, $G_{\mathrm{F}}$, and the corresponding tension softening diagram $\boldsymbol{\sigma}(w)$ relating the residual stress transfer capa-

*School of Engineering, Cardiff University, Queen's Buildings, PO Box 925, Cardiff CF24 0YF, UK.

(MCR 1235) Paper received 5 February 2004; last revised 14 June 2004; accepted 12 August 2004 city $\sigma$ to the opening displacement $w$ of the fictitious crack faces. In practice, the $\boldsymbol{\sigma}(w)$ relationship is often approximated by a linear, bilinear, polylinear or even exponential curve ${ }^{2}$ with the bilinear approximation being the most common. The popularity of the bilinear approximation of the tension softening diagram (TSD) stems from the fact that it captures the two major mechanisms responsible for the observed tension softening in concrete, namely microcracking and aggregate interlock. The initial, steep branch of the bilinear TSD is a result of microcracking, whereas the second, tail branch is a result of aggregate interlock.

The authors have recently proposed a simple method for the determination of the true $G_{\mathrm{F}}$ of a concrete mix that is independent of the shape and size of the test specimen. ${ }^{3,4}$ This method requires the determination of the specific fracture energy $G_{\mathrm{f}}(\alpha, W)$ on specimens of the same size $W$ but one half of which contain a very shallow starter notch $a(\alpha=a / W)$ and the other half a deep starter notch. The two specific fracture energy values so determined depend on $\alpha$ and $W$. The simplicity of the method lies in how the true specific fracture energy of the concrete mix $G_{\mathrm{F}}$ can be calculated from the two size-dependent values $G_{\mathrm{f}}(\alpha, W)$. It was shown ${ }^{4}$ that for the commonly used three-point bend (TPB) and wedge-splitting (WS) specimens the $\alpha$ values should be 0.05 and 0.50 for TPB, and 0.20 and 0.50 for WS specimens. 


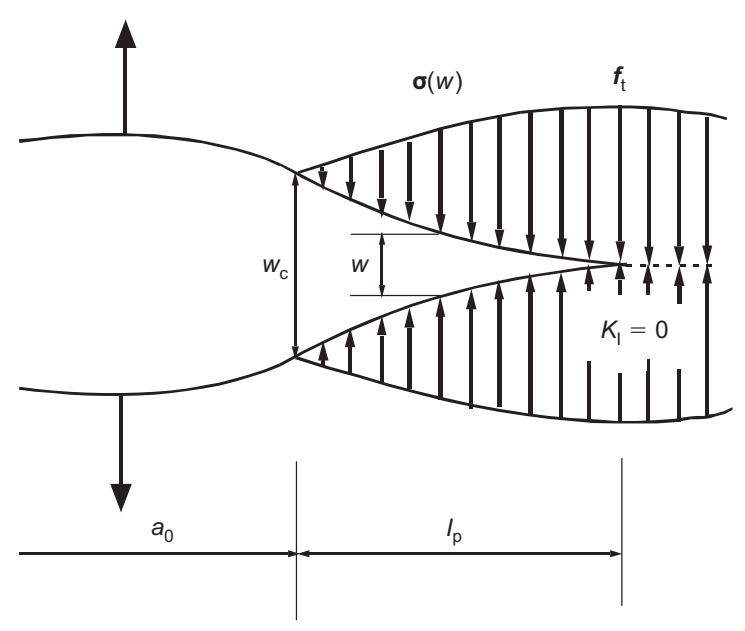

(a)

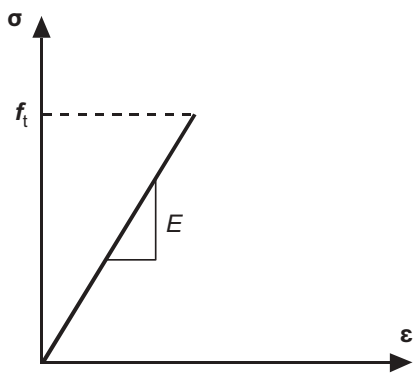

(b)

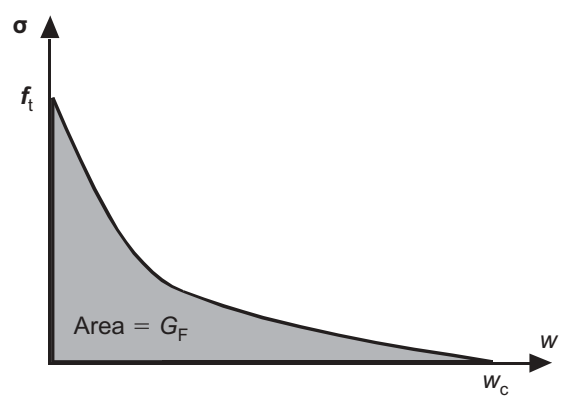

(c)

Fig. 1. (a) A real traction-free crack of length $a_{0}$ terminating in a fictitious crack of length $l_{\mathrm{p}}$ whose faces close smoothly near its tip $\left(K_{\mathrm{I}}=0\right)$. The material ahead of the fictitious crack tip is assumed to be linear (b), but the material within the fracture process zone is softening; the area under softening curve equals fracture energy $G_{\mathrm{F}}(c)\left(\right.$ after Karihaloo $\left.{ }^{2}\right)$.

The determination of TSD has proved to be a major problem and is still not a simple task to perform. Several researchers have attempted to measure the TSD using the direct tension test. ${ }^{5,6}$ However, this requires a special test set-up, and it is very difficult to obtain stable loading condition during the test. Moreover, the direct tension test measures the average stress-deformation response of the specimen and not the accurate relationship of cohesive stress and crack opening. ${ }^{7}$ Therefore, other researchers ${ }^{8,9}$ have focused on the indirect determination of TSD based on, for example, the determination of the load-displacement curve of a TPB specimen and a subsequent inverse analysis in which a finiteelement model forms the basis of the modelling.

An analytical model based on the concept of a nonlinear hinge was proposed, ${ }^{10}$ in which the flexural response of concrete beams was modelled by the development of a fictitious crack in the central region of the beam subjected to the maximum bending moment. The width of this region, proportional to the beam depth, fixes the width of the non-linear hinge. It was assumed that $\boldsymbol{\sigma}(w)$ is linear. This model was further developed by Stang and Olesen ${ }^{11}$ to demonstrate its applicability to TPB beams using a bilinear approximation for $\boldsymbol{\sigma}(w)$.
This paper will describe an inverse procedure also based on the non-linear hinge concept for identifying the parameters of the TSD corresponding to the true specific fracture energy $G_{\mathrm{F}}$. The TSD is assumed to be bilinear in shape.

\section{Hinge model}

The basic idea of the non-linear cracked hinge model is to isolate the part of the beam close to the propagating crack (i.e. the part under maximum bending moment) as a short beam segment subjected to a bending moment and normal force. Fig. 2 shows a typical TPB and a typical WS specimen.

In the non-linear hinge model the crack is viewed as a local change in the overall stress and strain field. This change is assumed to vanish outside a certain band of width $s$ (see Fig. 2). Thus, outside of this band the structural element is modelled using the elastic beam theory.

The constitutive relationship for each segment inside the hinge is assumed to be linear elastic in the precracked state (phase 0), while the cracked state is approximated by a bilinear softening curve, see Fig. 3,

Magazine of Concrete Research, 2004, 56, No. 10 


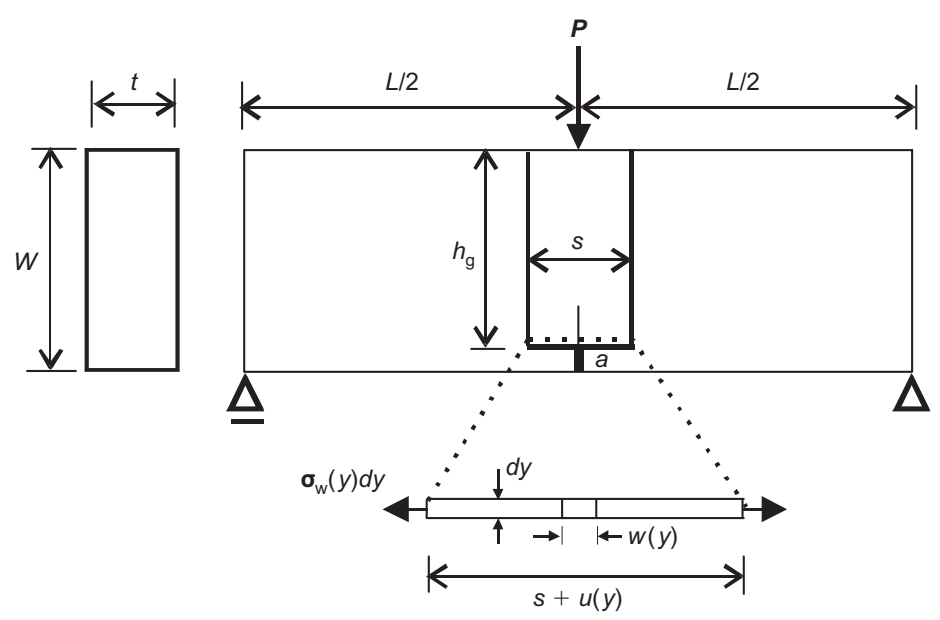

(a)

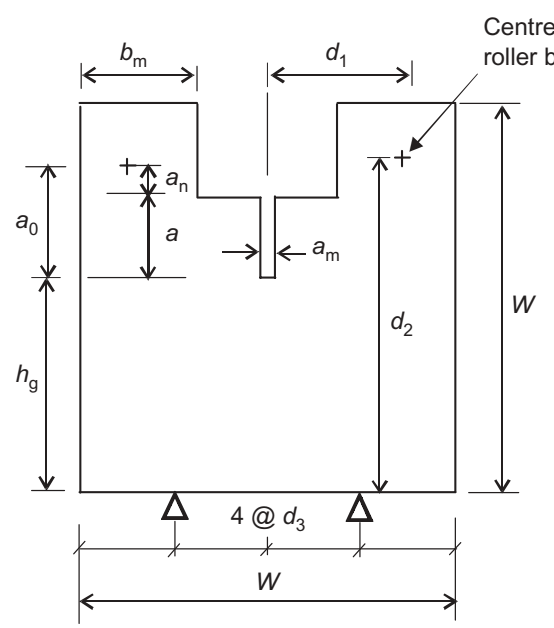

Centre of

oller bearings
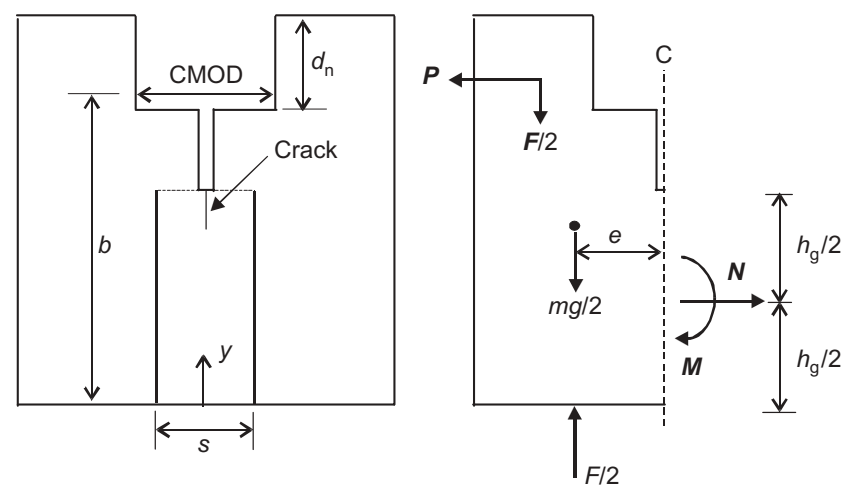

(b)

Fig. 2. Three-point notched bend beam with a non-linear hinge modelling the propagation of a crack at mid-section (a) and geometry and the loading of wedge splitting test specimen showing the hinge element and its loading (b)

$$
\boldsymbol{\sigma}= \begin{cases}E \boldsymbol{\varepsilon} & \text { pre-cracked state } \\ \boldsymbol{\sigma}(w)=g(w) f_{\mathrm{t}} & \text { cracked state }\end{cases}
$$

where $E$ is the elastic modulus; $\boldsymbol{\varepsilon}$ is the elastic strain; $w$ is the crack opening; $f_{\mathrm{t}}$ is the uniaxial tensile strength; and $g(w)$ is the function representing the shape of the stress-crack opening relationship, normalised such that $g(0)=1$. For the assumed bilinear shape (Fig. 3) we have

$$
\begin{gathered}
g(w)=b_{\mathrm{i}}-a_{\mathrm{i}} w=\left\{\begin{array}{l}
b_{1}-a_{1} w, 0 \leqslant w \leqslant w_{1} \\
b_{2}-a_{2} w, w_{1} \leqslant w \leqslant w_{2}
\end{array}\right. \\
w_{1}=\frac{1-b_{2}}{a_{1}-a_{2}} ; \\
w_{2}=\frac{b_{2}}{a_{2}},
\end{gathered}
$$

where $b_{1} \equiv 1$; and the limits $w_{1}$ and $w_{2}$ are given by the intersection of the two line segments, and the intersection of the second line segment with the abscissa, respectively (see Fig. 3).
The geometry of WS test specimens with $W=200$ $\mathrm{mm}$ is shown in more detail in Fig. 2(b) and Table 1. The size of the cube is $W$ and the initial notch length is $a_{0}$, while the ligament length of the hinge is $h_{\mathrm{g}}=W-d_{\mathrm{n}}-a_{0}$.

Analysis of the hinge element allows for the determination of the axial load $\boldsymbol{N}$ and bending moment $\boldsymbol{M}$ for any given hinge rotation $2 \varphi$ (see Fig. 4). The problem now is solved in four stages, one for each phase of crack propagation. Phase 0 represents the elastic state, when no crack has formed from the initial notch, while phases I, II and III represent different stages of crack propagation (see Fig. 3). In phase I, the fictitious crack of length $d$ is such that the maximum crack opening is less than $w_{1}$. In phase II, a part of the fictitious crack of length $d$ has a crack opening in excess of $w_{1}$ but in the remaining part it is less than $w_{1}$. In phase III, a part of the crack has opened more than $w_{2}$ and thus become traction-free, while the opening of the remaining part is still less than $w_{2}$ or even less than $w_{1}$. 


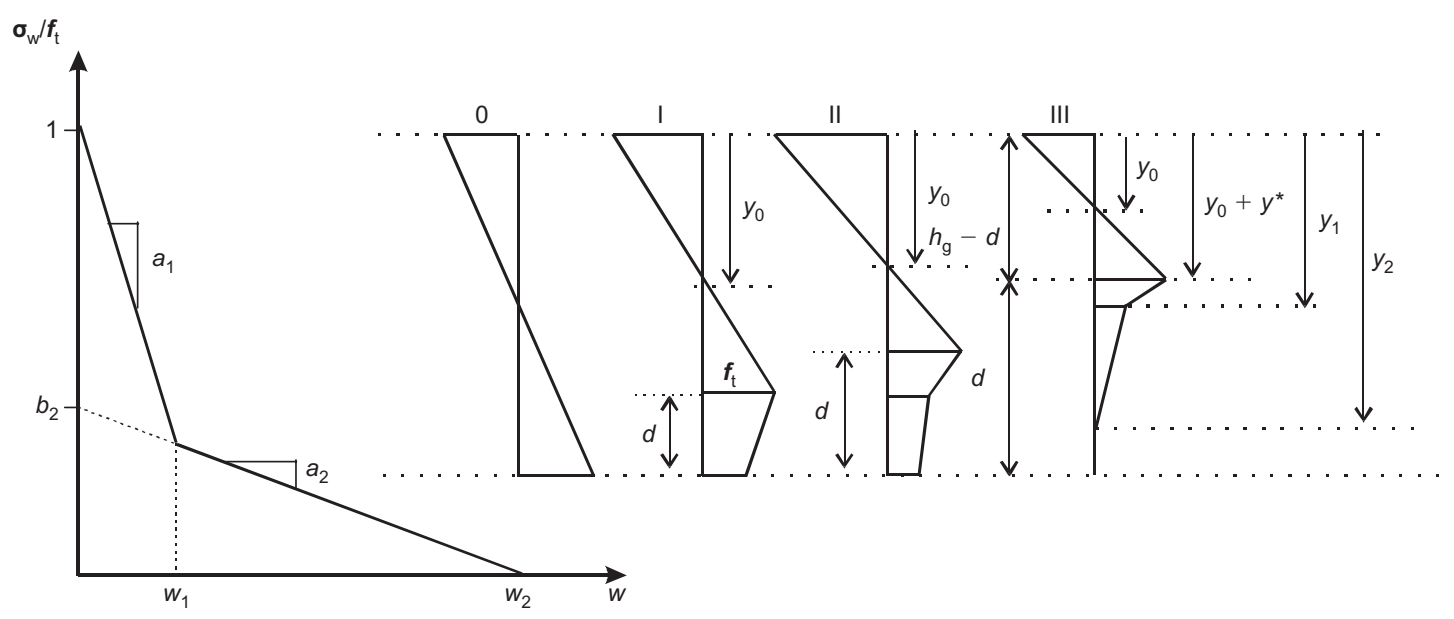

Fig. 3. Definition of a bilinear stress-crack opening relationship and the four different phases of crack propagation. Phase $0=$ state of stress prior to cracking; Phases I-III = states of stress during crack propagation.

Table 1. Dimensions of the $200 \mathrm{~mm}$ WS specimen ( $\mathrm{mm}$ )

\begin{tabular}{l|c|c|c|c|c|c|c|c|c|c}
\hline$W$ & $a$ & $a_{\mathrm{m}}$ & $a_{\mathrm{n}}$ & $b$ & $b_{\mathrm{m}}$ & $d_{1}$ & $d_{2}$ & $d_{3}$ & $d_{\mathrm{n}}$ & $h_{\mathrm{g}}$ \\
\hline 200 & $30-82 \cdot 5$ & 3 & 5 & 174 & 70 & 75 & 175 & 50 & 20 & $140-87 \cdot 5$ \\
\hline
\end{tabular}
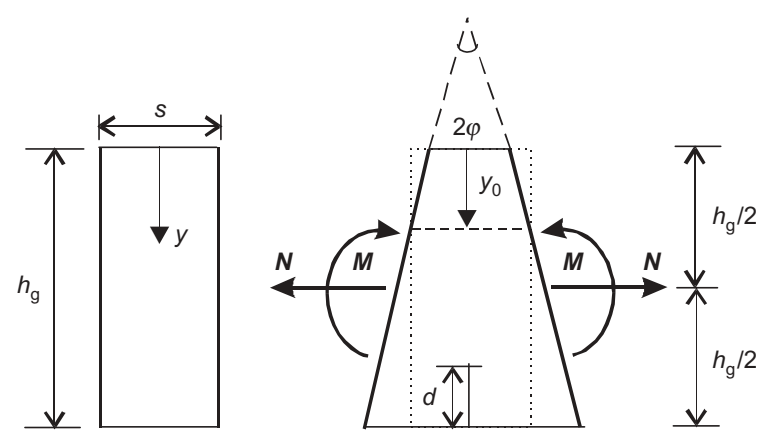

Fig. 4. Geometry, loading and deformation of the hinge element

When the complete stress distribution is established for the non-linear hinge, a relation between the normal force $\boldsymbol{N}$, the moment, $\boldsymbol{M}$ and the hinge rotation, $\varphi$ may be obtained in each phase of the crack propagation. The following normalised parameters are introduced

$$
\mu=\frac{6}{\boldsymbol{f}_{\mathrm{t}} h_{g}^{2} t} \boldsymbol{M} ; \rho=\frac{1}{\boldsymbol{f}_{\mathrm{t}} h_{\mathrm{g}} t} \boldsymbol{N} ; \theta=\frac{h_{\mathrm{g}} E}{s \boldsymbol{f}_{\mathrm{t}}} \varphi ; \alpha_{\mathrm{h}}=\frac{d}{h_{\mathrm{g}}}
$$

where $t$ is the width of the hinge in the direction normal to the paper and $d$ is the depth of the fictitious crack. Given these normalisations the pre-crack elastic behaviour of the hinge is described by $\alpha_{\mathrm{h}}=0$ and $\mu=$ $\theta$, where $0 \leqslant \theta<1-\rho$; at the onset of cracking $\theta=$ $1-\rho$. For TPB specimens $\rho=0$.

\section{Application of hinge model to TPB and wedge-splitting specimens}

The opening displacement at the mouth of the preexisting starter crack (CMOD) in TPB and WS specimens consists of three contributions. These are the opening due to the crack emanating from the starter crack, $\delta_{\mathrm{COD}}$, the opening due to elastic deformation, $\delta_{\mathrm{e}}$ and the opening due to geometrical considerations because the line of application of the load is shifted relative to the mouth of the starter crack, $\delta_{\mathrm{g}}$

$$
C M O D=\delta_{\mathrm{COD}}+\delta_{\mathrm{e}}+\delta_{\mathrm{g}}
$$

$\delta_{\mathrm{COD}}$ is the crack opening at the bottom of the crack in the hinge, that is, at $y=h_{\mathrm{g}}$, see Fig. 2 and 4 ,

$$
\delta_{\mathrm{COD}}=\frac{s \boldsymbol{f}_{\mathrm{t}}}{E} \frac{\left(1-b_{\mathrm{i}}+2 \alpha_{\mathrm{h}} \theta\right)}{\left(1-\beta_{\mathrm{i}}\right)}
$$

where $s$ is the width of the hinge, $\alpha_{\mathrm{h}}=d / h_{\mathrm{g}}$, $\theta=h_{\mathrm{g}} E \varphi / s \boldsymbol{f}_{\mathrm{t}}, \varphi$ is half of the hinge rotation and the parameter $\beta_{\mathrm{i}}$ can be obtained from

$$
\beta_{\mathrm{i}}=\frac{s a_{\mathrm{i}} f_{\mathrm{t}}}{E}
$$

$\delta_{\mathrm{e}}$ in equation (5) can be found from handbooks. ${ }^{12}$ The contribution from $\delta_{\mathrm{g}}$ has been found to be negligible for the specimen geometries tested.

The applied load in the TPB specimen is related to the normalised moment through the following relation

$$
\boldsymbol{P}=\frac{2}{3} \frac{\boldsymbol{f}_{\mathrm{t}} h_{g}^{2} t}{L} \mu(\theta)
$$

Magazine of Concrete Research, 2004, 56, No. 10 
where $L$ is the total length of the beam.

For the WS geometry no explicit expression for the load $\boldsymbol{P}$ in the cracked phase can be derived similar to equation (8) for the TPB specimen. For the pre-cracked phase (phase 0), the magnitude of the load, $\boldsymbol{P}^{0}$ is given by Reference 13

$$
\boldsymbol{P}^{0}=\frac{f_{\mathrm{t}} h_{g}^{2} t}{6 d_{2}-3 h_{\mathrm{g}}+3 k\left(d_{1}-d_{3}\right)}
$$

where, $k=2 \tan \alpha_{\mathrm{w}}$ depends on the wedge angle, $\alpha_{\mathrm{w}}$ ( $\alpha_{\mathrm{w}}=15^{\circ}$ for the $200 \mathrm{~mm}$ wedge-splitting geometry considered here).

In the cracked phases I-III, the solution for $\boldsymbol{P}$ can be obtained in iterative manner from the following implicit equation

$$
\mu_{\text {ext }}(\boldsymbol{P})-\mu_{\text {int }}^{j}(\theta, \boldsymbol{P})=0 .
$$

where $\mu_{\text {ext }}(\boldsymbol{P})$ represents the external loading on the hinge which can be determined from the force balance condition (see Fig. 1b), while $\mu_{\text {int }}^{j}(\theta, \boldsymbol{P})$ is the internal loading capacity of the hinge given by analytical expressions corresponding to the actual phase of the crack propagation. These expressions have been obtained by considering the force and moment equilibrium of the hinge in each crack propagation phase. ${ }^{11}$

For an additional check on the accuracy, $\boldsymbol{P}$ can be determined for a given value of CMOD from

$$
C M O D_{\exp }-C M O D(\boldsymbol{P}, \theta)=0
$$

where $C M O D_{\text {exp }}$ is the experimentally measured $C M O D$ and $C M O D(\boldsymbol{P}, \theta)$ is determined from equation (5).

The value of $\theta$ corresponding to each phase is calculated from the analytical expression. ${ }^{11}$ Then for this value of $\theta$ the normalised moment $\mu(\theta)$ and the crack length, $\alpha_{\mathrm{h}}$ are calculated, followed by the theoretical CMOD and load $\boldsymbol{P}$ (from equations (8), (9) and (10)). Next the sum of squares of the errors between the theoretical and experimental values of the load is minimised with respect to the three unknown parameters of the bilinear TSD

$$
\min _{\left(a_{1}, a_{2}, b_{2}\right)} \frac{1}{n} \sum_{0}^{n}\left(\boldsymbol{P}-\boldsymbol{P}_{\exp }\right)^{2}
$$

where $n$ is the total number of the observations representing the selected entries of $\theta$-that is, the selected values of $\boldsymbol{P}$ on the experimentally recorded loadCMOD diagram.

\section{Results and discussion}

Figures 5 and 6 show the mean of three or four load-CMOD diagrams recorded on $200 \mathrm{~mm}$ TPB and WS specimens, respectively, with two notch-to-depth ratios $(0.05,0.50$ for TPB and $0.20,0.50$ for WS)

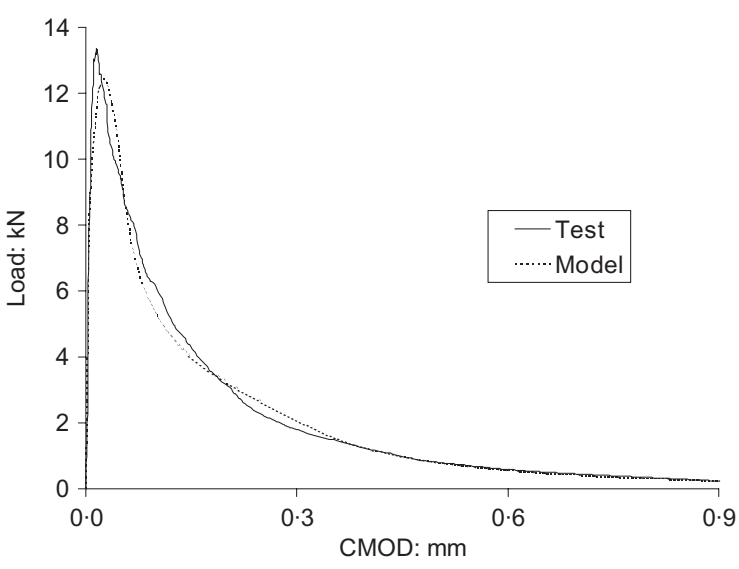

(a)

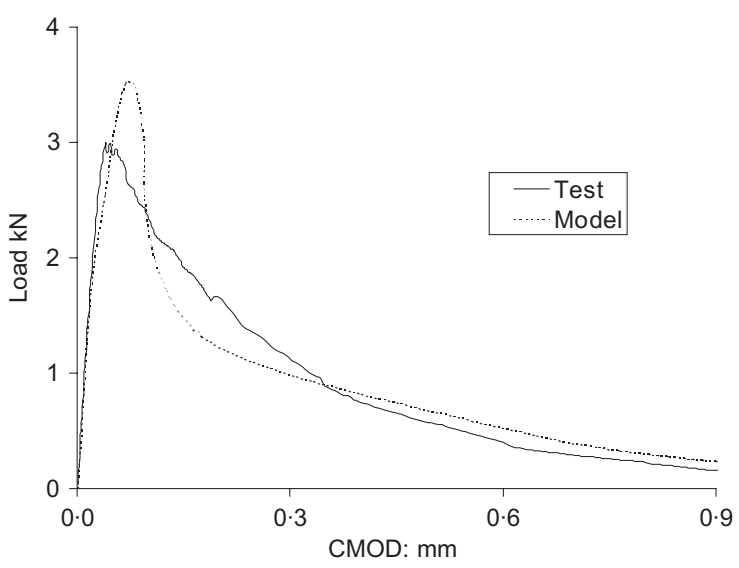

(b)

Fig. 5. Load-CMOD curves generated by the hinge model using the parameters obtained from the inverse analysis and the average experimental load-CMOD curves for TPB specimen size $200 \mathrm{~mm}$ with two notch to depth ratios (a) 0.05 and (b) $0 \cdot 50$

identified for the determination of the true $G_{\mathrm{F}}{ }^{4}$ The optimised coefficients $a_{1}, a_{2}$ and $b_{2}$ obtained by using the hinge model and minimising the difference between the theoretical and recorded load-CMOD curves are given in Table 2. The predictions from the hinge model corresponding to these parameters for each value of $\alpha$ are compared with the mean experimental results in Figs 5 and 6. The variations in the parameters with $\alpha$ are to be expected because of the variation in the measured $G_{\mathrm{f}}(\alpha)$.

\section{Parameters of bilinear TSD corresponding to $\mathbf{G}_{\mathbf{F}}$}

The procedure to obtain the true specific fracture energy $G_{\mathrm{F}}$ for a concrete mix from the size-dependent $G_{\mathrm{f}}(\alpha, W)$ measured in the laboratory was described in References 3 and 4. It is now necessary to establish the bilinear softening diagram corresponding to this $G_{\mathrm{F}}$. 


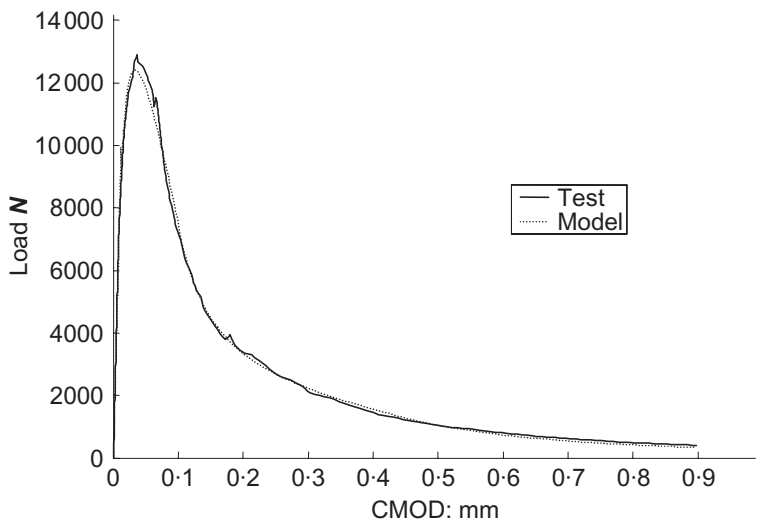

(a)

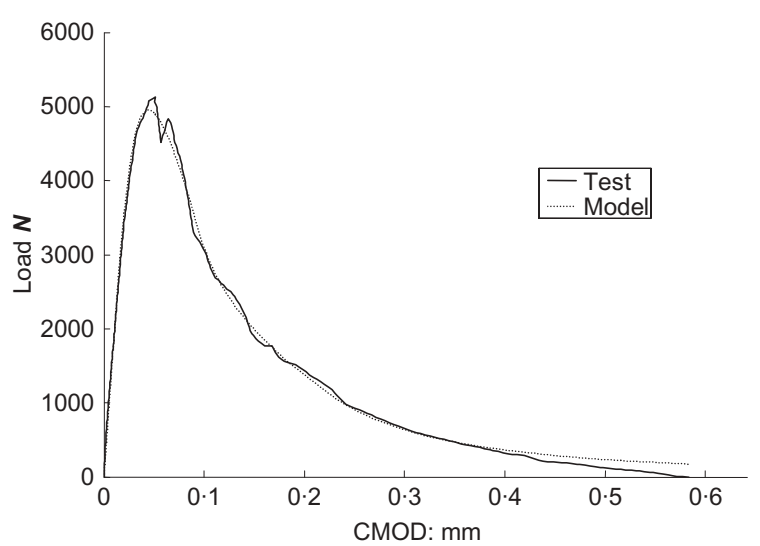

(b)

Fig. 6. The load-CMOD curve for $200 \mathrm{~mm}$ wedge splitting specimen compared with the load-CMOD curve generated by the hinge model for notch to depth ratio of (a) 0.20 and (b) 0.50 .

As described above, the parameters of the bilinear softening curve (Fig 7) were established by using the hinge model. The area under the softening curve obtained using the hinge model was equal not to $G_{\mathrm{F}}$ but to the measured $G_{\mathrm{f}}(\alpha, W)$, (see Table 2). It will be seen that the area under the bilinear TSD is generally less than the true $G_{\mathrm{F}}$. Thus the true $G_{\mathrm{F}}$ of the normal strength concrete used in TPB tests ${ }^{3}$ is $141 \cdot 1 \mathrm{~N} / \mathrm{m}$, whereas the average area under the bilinear TSD is only $129.1 \mathrm{~N} / \mathrm{m}$ (Table 2). Similarly, the true $G_{\mathrm{F}}$ of normal strength concrete used in WS tests ${ }^{3}$ is $154 \cdot 8$

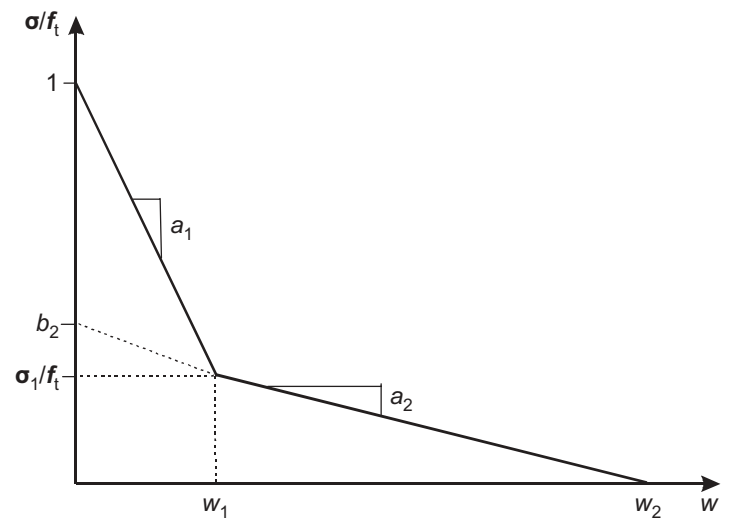

Fig. 7. Bilinear tension softening diagram.

$\mathrm{N} / \mathrm{m}$, whereas the average area under the bilinear TSD is only $133.5 \mathrm{~N} / \mathrm{m}$ (Table 2 ).

The size-dependent fracture energy (i.e. the area under the bilinear TSD) is given by

$$
G_{\mathrm{f}}^{*}(\alpha, W)=\frac{1}{2} \boldsymbol{f}_{\mathrm{t}}^{*}\left(w_{1}^{*}+\frac{\boldsymbol{\sigma}_{1}^{*}}{f_{\mathrm{t}}^{*}} w_{2}^{*}\right)
$$

where the superscript $*$ denotes the average parameters of the bilinear diagram obtained from the hinge model (Table 2).

The size-independent fracture energy (i.e. the area under the bilinear TSD corresponding to $G_{\mathrm{F}}$ ) can be similarly written as

$$
G_{\mathrm{F}}=\frac{1}{2} \boldsymbol{f}_{\mathrm{t}}\left(w_{1}+\frac{\boldsymbol{\sigma}_{1}}{\boldsymbol{f}_{\mathrm{t}}} w_{2}\right)
$$

where $w_{1}, w_{2}$ and $\boldsymbol{\sigma}_{1}$, which are to be determined, are the bilinear diagram parameters corresponding to the true fracture energy $G_{\mathrm{F}}$, and $\boldsymbol{f}_{\mathrm{t}}$ is the direct tensile strength of the mix obtained from an independent test, say a split cylinder test, $\boldsymbol{f}_{\mathrm{st}}$. It is assumed that $\boldsymbol{f}_{\mathrm{t}}=$ $0.65 \boldsymbol{f}_{\text {st. }}{ }^{14}$

The hinge model parameters corresponding to $G_{\mathrm{f}}$ $(\alpha, W)$ are now scaled to the true fracture energy $G_{\mathrm{F}}-$ that is

Table 2. Bilinear softening relationship parameters for normal strength concrete NC generated from the hinge model for $200 \mathrm{~mm}$ three-point bend and WS specimens for two different notch to depth ratios.

\begin{tabular}{l|r|r|r|r|r|r}
\hline \multirow{2}{*}{ Notch-to-depth ratio, $\alpha$} & \multicolumn{3}{|c|}{ TPB } & \multicolumn{3}{c}{ WS } \\
\cline { 2 - 7 } & \multicolumn{1}{|c|}{$0 \cdot 05$} & 0.50 & Average & $0 \cdot 20$ & \multicolumn{1}{c}{$0 \cdot 50$} & Average \\
\hline$a_{1}\left(\mathrm{~mm}^{-1}\right)$ & $20 \cdot 850$ & $43 \cdot 120$ & $27 \cdot 480$ & $11 \cdot 515$ & $14 \cdot 245$ & $12 \cdot 880$ \\
$a_{2}\left(\mathrm{~mm}^{-1}\right)$ & $0 \cdot 600$ & $0 \cdot 600$ & $0 \cdot 600$ & $1 \cdot 608$ & $1 \cdot 196$ & $1 \cdot 402$ \\
$b_{2}(\mathrm{~mm})$ & $0 \cdot 185$ & $0 \cdot 187$ & $0 \cdot 186$ & $0 \cdot 425$ & $0 \cdot 343$ & $0 \cdot 384$ \\
$w_{1}(\mathrm{~mm})$ & $0 \cdot 040$ & $0 \cdot 024$ & $0 \cdot 030$ & $0 \cdot 058$ & $0 \cdot 050$ & 0.054 \\
$w_{2}(\mathrm{~mm})$ & $0 \cdot 308$ & $0 \cdot 311$ & $0 \cdot 310$ & $0 \cdot 264$ & $0 \cdot 283$ & $0 \cdot 274$ \\
$G_{\mathrm{f}}(\mathrm{N} / \mathrm{m})$ & $141 \cdot 100$ & $122 \cdot 200$ & $129 \cdot 100$ & $116 \cdot 500$ & $150 \cdot 500$ & $133 \cdot 500$ \\
\hline
\end{tabular}




$$
\frac{1}{2} \boldsymbol{f}_{\mathrm{t}}\left(w_{1}+\frac{\boldsymbol{\sigma}_{1}}{\boldsymbol{f}_{\mathrm{t}}} w_{2}\right)=\frac{1}{2} \boldsymbol{f}_{\mathrm{t}}^{*}\left(w_{1}^{*}+\frac{\boldsymbol{\sigma}_{1}^{*}}{\boldsymbol{f}_{\mathrm{t}}^{*}} w_{2}^{*}\right) \frac{G_{\mathrm{F}}}{G_{\mathrm{f}}^{*}(\alpha, W)}
$$

The coordinates of the knee of the bilinear diagram predicted by the hinge model are related as follows (Fig. 7)

$$
\frac{\boldsymbol{\sigma}_{1}^{*}}{\boldsymbol{f}_{\mathrm{t}}}=1-a_{1}^{*} w_{1}^{*}
$$

A term-by-term comparison of the two sides of equation (15) gives

$$
\begin{gathered}
w_{1}=w_{1}^{*} \frac{G_{\mathrm{F}}}{G_{\mathrm{f}}^{*}} \frac{f_{\mathrm{t}}^{*}}{f_{\mathrm{t}}} \\
\frac{\boldsymbol{\sigma}_{1}}{\boldsymbol{f}_{\mathrm{t}}}=\frac{G_{\mathrm{F}}}{G_{\mathrm{f}}^{*}} \frac{\boldsymbol{\sigma}_{1}^{*}}{\boldsymbol{f}_{\mathrm{t}}^{*}} \frac{w_{2}^{*}}{w_{2}} \frac{\boldsymbol{f}_{\mathrm{t}}^{*}}{\boldsymbol{f}_{\mathrm{t}}}
\end{gathered}
$$

From Fig. 7 we obtain an additional equation for the slope $a_{2}$ of the true bilinear diagram

$$
\frac{\boldsymbol{\sigma}_{1}}{\boldsymbol{f}_{\mathrm{t}}}=\left(w_{2}-w_{1}\right) a_{2}
$$

Equating the two equations (18) and (19) gives a quadratic equation for calculating the crack opening, $w_{2}$

$$
w_{2}^{2}-w_{1} w_{2}=\frac{1}{a_{2}} \frac{G_{\mathrm{F}}}{G_{\mathrm{f}}^{*}} \frac{\boldsymbol{\sigma}_{1}^{*}}{\boldsymbol{f}_{\mathrm{t}}} w_{2}^{*}
$$

once $w_{1}$ has been determined from equation (17). Note that slope $a_{2}$ is chosen to coincide with $a_{2}^{*}$ of TPB specimen, that is it is assumed that the slope of the tail part of the bilinear diagram is not sensitive to $\alpha$ and $W$. This is a reasonable assumption in view of the fact that $a_{2}$ is a result of the aggregate interlock which is primarily governed by the maximum size and texture of the coarse aggregate used in the concrete mix.

The parameters of the bilinear TSD corresponding to the true $G_{\mathrm{F}}$ values of the two concrete mixes used in this study are given in Table 3. The TSDs are plotted in Fig. 8. It is worth mentioning that the two normalstrength concrete mixes are very similar in their mechanical properties. This remains true of their bilinear TSDs too, despite the fact that these were inferred by inverse analysis on TPB and WS test data. This gives

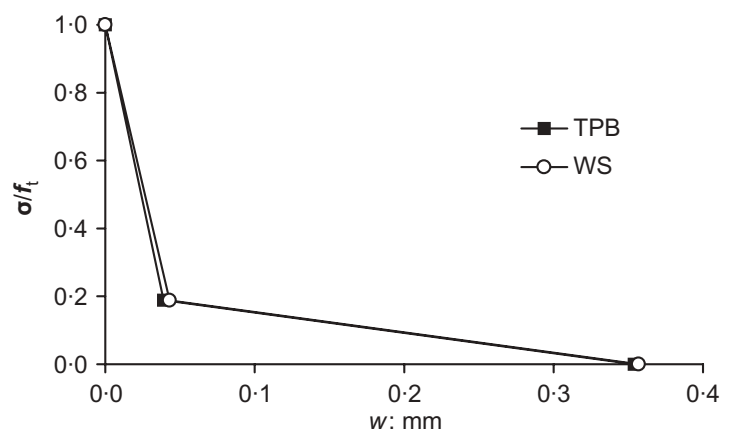

Fig. 8. The normalised bilinear stress-crack opening relationship for two normal-strength concretes corresponding to their true fracture energy $G_{\mathrm{F}}$.

confidence in the predictions of the non-linear hinge model for inverse analysis.

\section{Conclusions}

For the analysis of cracked concrete structures using the non-linear theory of fracture mechanics based on the fictitious crack model, two fracture properties of concrete are needed, namely the specific fracture energy $G_{\mathrm{F}}$ and the corresponding tension softening relation $\boldsymbol{\sigma}(w)$. The authors have recently proposed a simple method for the determination of the true $G_{\mathrm{F}}$ of concrete mix from the specific fracture energy $G_{\mathrm{f}}(\alpha, W)$ measured on TPB or WS specimens of the same dimension $W$ but containing starter cracks $\alpha$ which are well separated.

In this paper, it has been shown how the parameters of a bilinear approximation of the tension softening relation $\boldsymbol{\sigma}(w)$ corresponding to the recorded loadCMOD diagram of the TPB and WS specimens can be inferred through an inverse analysis based on the concept of a non-linear hinge. The parameters so inferred will also depend on the shape and size of the test specimen, just as the specific fracture energy $G_{\mathrm{f}}(\alpha, W)$. A procedure has been proposed to scale these parameters so that they correspond to the true $G_{\mathrm{F}}$ of the concrete mix that is independent of the shape and size of the test specimen. Thus the two fracture properties of concrete needed for the analysis of cracked concrete structures can be obtained from simple tests and analysis of the

Table 3. Elastic properties and parameters of the bilinear softening diagram corresponding to the size-independent fracture energy $G_{\mathrm{F}}$ for the two concrete mixes.

\begin{tabular}{l|c|c|c|c|c|c|c|c}
\hline Mix & $\begin{array}{c}f_{\mathrm{c}} \\
\mathrm{MPa}\end{array}$ & $\begin{array}{c}f_{\mathrm{t}} \\
\mathrm{MPa}\end{array}$ & $\begin{array}{c}G_{\mathrm{F}} \\
\mathrm{N} / \mathrm{m}\end{array}$ & $\begin{array}{c}E \\
\mathrm{Gpa}\end{array}$ & $\begin{array}{c}w_{1} \\
\mathrm{~mm}\end{array}$ & $\begin{array}{c}w_{2} \\
\mathrm{~mm}\end{array}$ & $\begin{array}{c}\sigma_{1} \\
\mathrm{fm}_{\mathrm{t}} \\
\mathrm{MPa}\end{array}$ \\
\hline NSC for TPB & 55 & $2 \cdot 67$ & 141 & $36 \cdot 9$ & $0 \cdot 039$ & $0 \cdot 354$ & $20 \cdot 75$ \\
NSC for WS & 60 & $2 \cdot 80$ & 155 & $38 \cdot 3$ & $0 \cdot 043$ & $0 \cdot 357$ & $0 \cdot 189$ \\
\hline
\end{tabular}

$\mathrm{NSC}=$ normal strength concrete. 
test data using the procedures described above and in Reference 4.

\section{References}

1. Hillerborg A., Modeer M. and Petersson P. E. Analysis of crack formation and crack growth in concrete by means of fracture mechanics and finite elements. Cement and Concrete Research, 1976, 6, 773-782.

2. Karihaloo B. L. Fracture Mechanics and Structural Concrete, Addison Wesley Longman, UK, 1995.

3. Abdalla H. M. and Karihaloo B. L. Determination of sizeindependent specific fracture energy of concrete from threepoint bend and WS tests. Magazine of Concrete Research, 2003, 55, No. 2, 133-141.

4. Karihaloo B. L., Abdalla H. M. and Imjai T. A simple method for determining the true specific fracture energy of concrete. Magazine of Concrete Research, 2003, 55, No. 5, $471-481$.

5. Gopalaratnam V. S. and Shah S. P. Softening response of plain concrete in direct tension. ACI Journal, 1985, 310-323.

6. WANG Y., LI V. C. and BACKER S. Experimental determination of tensile behaviour of fiber reinforced concrete. ACI Journal, 1990, 87, 461-468.

7. Kitsutaka Y. Fracture parameters by polylinear tensionsoftening analysis. ASCE Journal of Engineering Mechanics, 1997, 123, 444-450.
8. Roelfstra P. E. and Wittmann F. H. Numerical method to link strain softening with failure of concrete. In Fracture Toughness and Fracture Energy of Concrete (edited by F. H. Wittmann), Elsevier, Amesterdam, The Netherlands, 1986, 163-175.

9. Wittmann F. H., Roelfstra P. E., Mihashi H., Huang Y. and ZHANG X. H. Influence of age of loading, water-cement ratio and rate of loading on fracture energy of concrete. Materials and Structures, 1987, 20, 103-110.

10. Ulfkjaer J. P., Krenk S. and Brincker R. Analytical model for fictitious crack propagation in concrete beams. ASCE Journal of Engineering Mechanics, 1995, 121, 7-15.

11. Stang H. and Olesen J. F. On the interpretation of bending tests on FRC-materials. Proceedings of FRAMCOS-3, Fracture Mechanics of Concrete Structures. Aedificatio Publishers. Freiburg, Germany, 1998, vol I, 511-520.

12. Tada H., Paris P. C. and Irwin G. R. The Stress Analysis of Cracks Handbook, Del Research Corp., Hellertown, PA, 1985.

13. Østergand L. Early-age Fracture Mechanics and Cracking of Concrete. PhD thesis, The Technical University of Denmark, Lyngby, 2003.

14. Neville A. M. Properties of Concrete 4th Edn., Longman Scientific, London, 1995, p. 844.

Discussion contributions on this paper should reach the editor by 1 June 2005 